\title{
Scanning Electrochemical Microscopy as a Characterization Tool for Reduced Graphene Oxide Field Effect Transistors
}

\author{
Ciril Reiner-Rozman* and Jürgen Schodl ${ }^{\dagger}$ \\ CEST Centre of Electrochemical Surface Technology, \\ Viktor-Kaplan-Strasse 2, Wiener Neustadt 2700, Austria \\ Christoph Nowak \\ CEST Centre of Electrochemical Surface Technology, \\ Viktor-Kaplan-Strasse 2, Wiener Neustadt 2700, Austria, and \\ AIT Austrian Institute of Technology, Muthgasse 11, Vienna 1190, Austria \\ Christoph Kleber \\ CEST Centre of Electrochemical Surface Technology, \\ Viktor-Kaplan-Strasse 2, Wiener Neustadt 2700, Austria \\ (Received 18 March 2015; Accepted 5 June 2015; Published 14 July 2015)
}

\begin{abstract}
Reduced graphene oxide coated $\mathrm{SiO}_{2} / \mathrm{Si}$ substrates were obtained by wet-chemical reduction of graphene oxide for the use as semiconductor material in field-effect transistors. The morphological and chemical characterization was done by using SEM, Raman spectroscopy and XPS. Raman and XPS measurements can characterize the success of the graphene-oxide reduction, but only for small parts spots of the surface (e.g. $0.41 \mu \mathrm{m}^{2}$ laser spot size with Raman). In order to evaluate larger surface areas and the electrochemical activity of the graphene oxide and reduced graphene oxide, additional spectroscopic measurements using the SECM were performed. The samples coated with unreduced graphene oxide showed no electrochemical activity, while reduced graphene oxide samples showed conducting properties. Further information about the topology of the surface was obtained by applying the SECM constant distance mode. The degree of graphene coverage was calculated from SECM data and compared to the coverage obtained by SEM. It was found that $68 \pm 7 \%$ coverage is sufficient to ensure electronic contact between the Source and Drain electrodes (resistance less than $1 \mathrm{k} \Omega$ ). Functionality of the fabricated field effect transistors was demonstrated by titration of $\mathrm{pH}$ solutions and characterization of the characteristic curves. [DOI: $10.1380 /$ ejssnt.2015.366]
\end{abstract}

Keywords: Scanning electrochemical microscopy; Ion-solid interactions; Field effect; Raman scattering spectroscopy; X-ray photoelectron spectroscopy; Conductivity; SECM; rGO FET

\section{INTRODUCTION}

Graphene is a two-dimensional, $\mathrm{sp}^{2}$-hybridized nanomaterial that can be synthesized from graphite [1] and has been examined over the last years on experimental and theoretical basis [2,3]. Graphene is often found in applications like biosensors [4], photonics [5], solar cells [6], medical use [7], superconducting materials [8] or as transparent electrodes [9]. Graphene exhibits diverse properties [10], depending on the fabrication process (e.g. scotch tape method, chemical vapour deposition (CVD) or wetchemical synthesis). These variable properties have a significant impact on the output characteristics of sensing devices fabricated for biosensing applications.

Although wet-chemically synthesized graphene has several advantages, including high yields and low production costs, problems with reduced graphene oxide (rGO) are the uncertainty of reduction success and the random surface coverage of graphene flakes. Therefore any method characterizing the surface coverage and the degree of reduction success of the GO sheets over a specified area (e.g., detection channel) is ideal to make statements about the statistical quality of fabricated sensing devices, triggering the interest in SECM (surface electrochemical microscope) characterization. Although methods like Ra-

\footnotetext{
* Corresponding author: Rozman.Ciril.fl@ait.ac.at

† Corresponding author: Juergen.Schodl@cest.at
}

man analysis or XPS offer options for analysis of reduction rate by analysis of binding energies of the material or for quantitative characterization of the reduction success by examining the amount of $s p^{2}$-hybridized orbitals, with these techniques only a very small part (spot) of the actual surface covered with rGO flakes is examined. A different approach to evaluate the degree of reduction of GO sheets is the application of SECM, measuring the electrochemical activity and sheet distribution at once for a larger area (up to a few $\mathrm{mm}^{2}$ ) $[11,12]$.

In general SECM has already been used as powerful technique to test the electrochemical activity of various surfaces, especially rGO coated surfaces [11, 12]. Furthermore, several applications in different research fields such as metals corrosion studies [13, 14], materials science [15], energy storage [16] or biological systems [17, 18] use the SECM as an established system. The advantage of the constant distance mode, used in this work, is the decoupling of the electrochemical activity information from the topography $[19,21]$.

In this work graphene-coated samples were obtained by reducing GO applied on the $\mathrm{SiO}_{2}$ substrates for the fabrication of field effect transistors (FETs). Raman and XPS were used for chemical characterization, while Scanning electron microscopy (SEM) was applied to observe the morphology of the rGO-coated surface. Furthermore the electronic properties of these FETs were tested in liquidgated as well as back-gated configuration and the sensing capability of the devices was demonstrated by $\mathrm{pH}$ titration. SECM measurements were performed to test 
and compare the electrochemical activity of the surface with the results from the mentioned methods. The SECM measurements were used for a simulated implementation of FET electrodes of $4 \mathrm{~mm}$ length [20]. Based on these results the rGO-connection in the transistor channel was evaluated to proof the statistical reproducibility of the FET fabrication process in spite of the random rGO distribution after application, bypassing the disadvantages of wet-chemically synthesized graphene.

\section{EXPERIMENTAL}

\section{A. FET preparation}

All measurements were carried out at room temperature. Graphene oxide (GO) was synthesized according to Larisika et al. [22] and FET fabricated according to [20]. Electrical properties were monitored using a Keithley semiconductor parameter analyzer, model 4200-SCS.

Solutions were prepared with $\mathrm{pH}$ variation of $0.1 \mathrm{pH}$ for the individual steps and injections were performed after equilibrium was established for the previous solutions (constant baseline slope). Current leakage through the solution channel was prevented using FET devices with very high channel conductivity, theoretically ensuring $99 \%$ of current flow through the rGO channel when calculated for the model of a parallel circuit.

\section{B. SEM, Raman \& XPS}

The investigation of the surface by scanning electron microscopy (SEM) was performed using a SUPRA 40 (Field emission scanning electron microscope, Carl Zeiss, Germany) with an acceleration voltage of $5 \mathrm{kV}$ and the magnification was 500x.

Raman measurements were performed using a Horiba LabRAM HR Raman confocal microscope (with a laser wavelength of $532 \mathrm{~nm}(52 \mathrm{~mW}, 10 \%$ filter, $200 \mu \mathrm{m}$ slit, $600 \mu \mathrm{m}$ hole, 100x objective) and a laser spot size of 0.41 $\left.\mu \mathrm{m}^{2}\right)$. Raman spectra were acquired using $2 \mathrm{~s}$ and $3 \mathrm{x}$ integration time.

X-ray Photoelectron Spectroscopy (XPS) analysis was performed on a VG ESCALAB 220i-XL with an Al Ka $(1486.6 \mathrm{eV})$ monochromatic X-ray source. Measured binding energy error is estimated to be within $\pm 0.2 \mathrm{eV}$.

\section{Scanning electrochemical microscopy}

A scanning electrochemical microscope system ElProScan ELP1, provided by HEKA Elektronik Dr. Schulze GmbH, Lambrecht/Pfalz, Germany (HEKA) was used. The system is equipped by a Controller Unit (ElProScan ESC 3), a bipotentiostat/galvanostat (PG 340 USB), a closed-loop optical-encoder based XYZ positioning system (Applied Scientific Instrumentation), a software controlled $45^{\circ}$ imaging system (HEKA) for prepositioning of the electrode tip on the sample under visual control, a shear force unit and a data acquisition software (Potmaster, HEKA). A figure of the main compo- nents of the SECM instrument is given in the supplemental information. Measurements were accomplished in a three-electrode configuration cell consisting of a Pt-disk microelectrode, a $\mathrm{Pt}$ wire auxiliary electrode and $\mathrm{Ag}$ wire with deposited $\mathrm{AgCl}$ as quasi-reference electrode. Ferrocenemethanol, $1 \mathrm{mM}$ in aqueous solution, was used as mediator (purity 97\%, Sigma Aldrich). Potassium chloride, $100 \mathrm{mM}$ in aqueous solution, (AnalaR NORMAPUR, 99.6\%, VWR Chemicals) was used as supporting electrolyte. Ultrapure water was obtained from a Seralpur Pro $90 \mathrm{CN}$ (Seral) system with conductivity values lower than $0.05 \mu \mathrm{Scm}^{-1}$. Pt-disk microelectrodes (tip) were purchased from HEKA. The tips were made of $10 \mu \mathrm{m}$ diameter $\mathrm{Pt}$ wire and were polished using a $0.05 \mu \mathrm{m}$ alumina polishing foil and then investigated by scanning electron microscopy (SEM) in means of a XL 30 ESEM-FEG (Environmental scanning electron microscope with field emission gun, Philips, Netherlands). The functionality was tested in aqueous solution of $1 \mathrm{mM}$ ferrocenemethanol $+100 \mathrm{mM} \mathrm{KCl}$ at HEKA. It can be seen from CV-measurements (Fig. S4 in Supplementary Materials) that the limiting diffusion current of the oxidation reaction of the ferrocenemethanol mediator begins at potential values higher than $200 \mathrm{mV}$ (vs. pseudo $\mathrm{Ag} / \mathrm{AgCl}$ reference electrode). Therefore the tip was positively polarized at a potential of $+300 \mathrm{mV}$ (vs. pseudo $\mathrm{Ag} / \mathrm{AgCl}$ reference electrode) to oxidize the reduced form of the redox mediator (Red) at a diffusion controlled rate $\left(i_{\infty}\right)$. The substrate surfaces in all the measurements were at their open circuit potential (OCP).

For the investigations of all surfaces the constant distance mode was used to scan a sample size up to $(350 \times$ 350) $\mu \mathrm{m}$. To maintain a constant distance between tip and sample surface a non-optical shear force method was used according to Schuhmann and co-workers [23-25, 33]. At the constant distance mode measurements the tip approach was stopped at a change of $50 \mathrm{mV} \mathrm{SF-amplitude}$ signal ( $\Delta \mathrm{SF}$-amplitude). Thus the distance between tip and surface was $60 \mathrm{~nm}$, independent from the topography of the sample. The frequency was $660.2 \mathrm{kHz}$ and applied voltage on dither piezo was $300 \mathrm{mV}$. After approaching the tip, the current was recorded, the tip pulled back $3 \mu \mathrm{m}$ from the surface (pull back distance) and then moved to the next measuring point. The surface was scanned with a resolution of $5 \mu \mathrm{m}$ in $x$ and $y$ direction. The recorded data were processed as $2 \mathrm{D}$ and $3 \mathrm{D}$ plots using the Software Mathlab (Mathworks, 7.13, R2011b) afterwards.

\section{RESULTS AND DISCUSSIONS}

\section{A. Scanning electron microscopy}

Topography of the deposited GO on the substrate was checked by SEM. Surface coverage was confirmed to be around $70 \%$, showing the majority of the surface being covered by monolayer rGO with a significant amount of double layered and multi-layered rGO as well (Fig. 1 (c)). The size of the rGO flakes varies between a few microns and up to approximately $1000 \mu \mathrm{m}^{2}$. Sheet thickness can be approximated from single layer graphene to a few layers. The empty spots found in SEM analysis can safely be seen as blank silicon due to it's flatness which would 


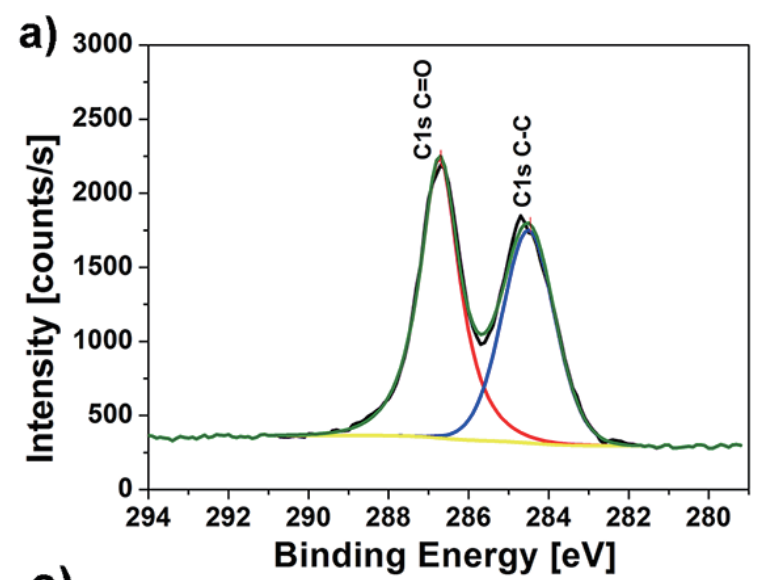

c)

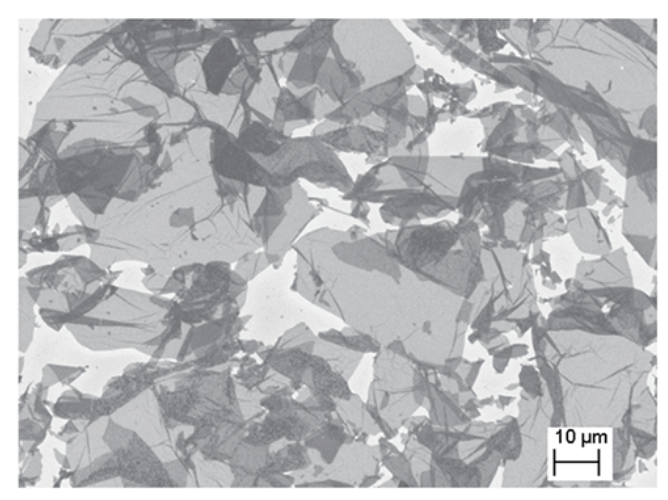

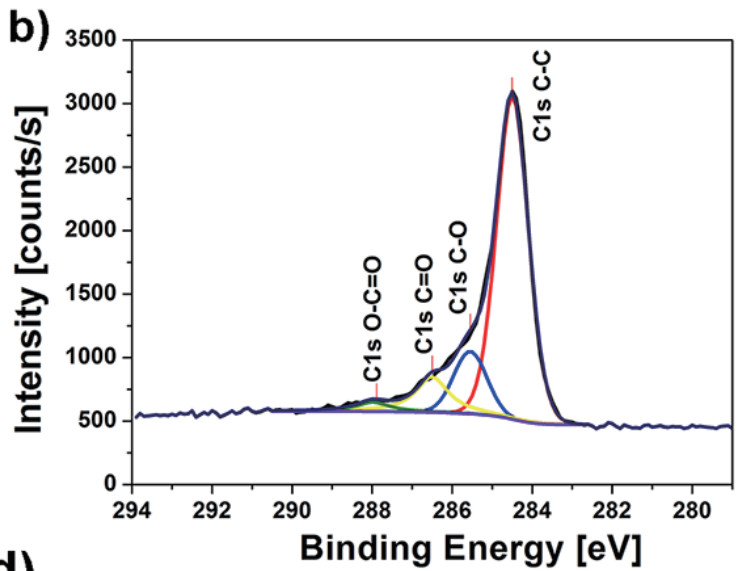

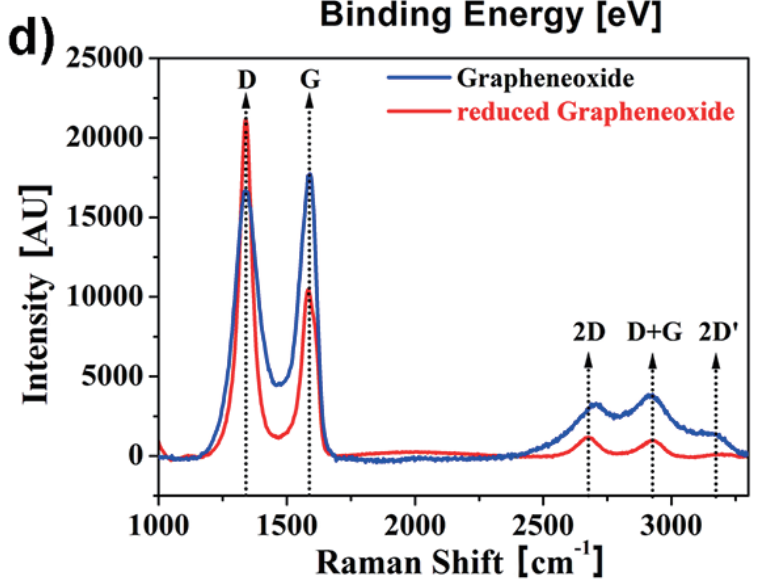

FIG. 1. a) XPS spectrum of unreduced GO. b) XPS spectrum of GO after reduction (rGO). The decline in intensity of the $\mathrm{C}=\mathrm{O}$ bond at $287 \mathrm{eV}$, to less than $5 \%$ of the value obtained before reduction, indicates reduction success. c) SEM image of rGO flake distribution after application via drop casting on $\mathrm{SiO}_{2} / \mathrm{Si}$-substrate; white areas: $\mathrm{SiO}_{2} / \mathrm{Si}$ substrate; light grey areas: rGO single-layers; dark grey areas: overlapped rGO single-layers. d) Raman spectra [532 nm] of GO-sample and rGO-sample; a comparison indicates successful reduction to rGO.

not correspond to a basal layers of graphene below the observed graphene clusters.

\section{B. XPS characterization}

X-ray Photoelectron Spectroscopy (XPS) measurements were done on GO as well as on rGO coated surfaces. The C1s spectrum of GO (Fig. 1 (a)) indicates the presence of two types of carbon bonds: C-C (284.5 $\mathrm{eV})($ composition $=46.5 \%), \mathrm{C}=\mathrm{O}(286.7 \mathrm{eV})($ composition $=53.5 \%)$. After hydrazine treatment (Fig. $1(\mathrm{~b}))$, the peak intensity of the $\mathrm{C}=\mathrm{O}$ (composition $=10.7 \%)$ species is much smaller than in the spectrum of GO, suggesting considerable de-oxygenation by the reduction process $[22$, $35]$.

Moreover, the composition of C-C increased to $72.3 \%$ in rGO $(284.5 \mathrm{eV})$. These values are consistent with reports from literature regarding the reduction of GO $[22,34]$. These experiments showed the decrease of the $\mathrm{O} 1 \mathrm{~s} / \mathrm{C} 1 \mathrm{~s}$ ratio and the increase of the $\mathrm{sp}^{2}$ hybridization.

\section{Raman spectroscopy}

Figure1 (d) shows the Raman spectra of GO and rGO in comparison. Both a graphitic-band (G-band, 1590 $\mathrm{cm}^{-1}$ ) and a second-order defect-band (2D-band, $\sim 2675$ $\mathrm{cm}^{-1}$ ) are observed in GO and rGO respectively which is in good agreement with the literature [26]. The observation of the D-band of GO and rGO at $\sim 1340 \mathrm{~cm}^{-1}$ suggests the presence of disordered structural defects from the attachment of oxygen containing groups on the carbon plane $[26,27]$. The results are in good agreement with the results obtained from XPS and those reported elsewhere [22, 28, 34], indicating successful reduction to rGO.

\section{Measurements of characteristic FET curves}

The applicability of the fabricated rGO as semiconducting thin-film electrodes was tested by fabrication of backgated FETs and measurements of the transfer characteristics. Figure 2 (a) shows the output characteristics (drain current $I_{\mathrm{d}}$ vs. drain voltage $V_{\mathrm{d}}$ ) at eleven different gate voltages $\left(V_{\mathrm{g}}\right)$, indicating increasing conductance with increasing gate voltage from $-100 \mathrm{~V}$ to $+100 \mathrm{~V}$. Hence the rGO films behave as p-type semiconducting materials [29]. Figure 2 (b) shows the dependence of $I_{\mathrm{d}}$ to a sweep of $V_{\mathrm{g}}$ at fixed $V_{\mathrm{d}}=0.1 \mathrm{~V}$. The charge neutrality point is found for positive gate voltages due to p-doping from persistent moisture and oxygen [27]. 

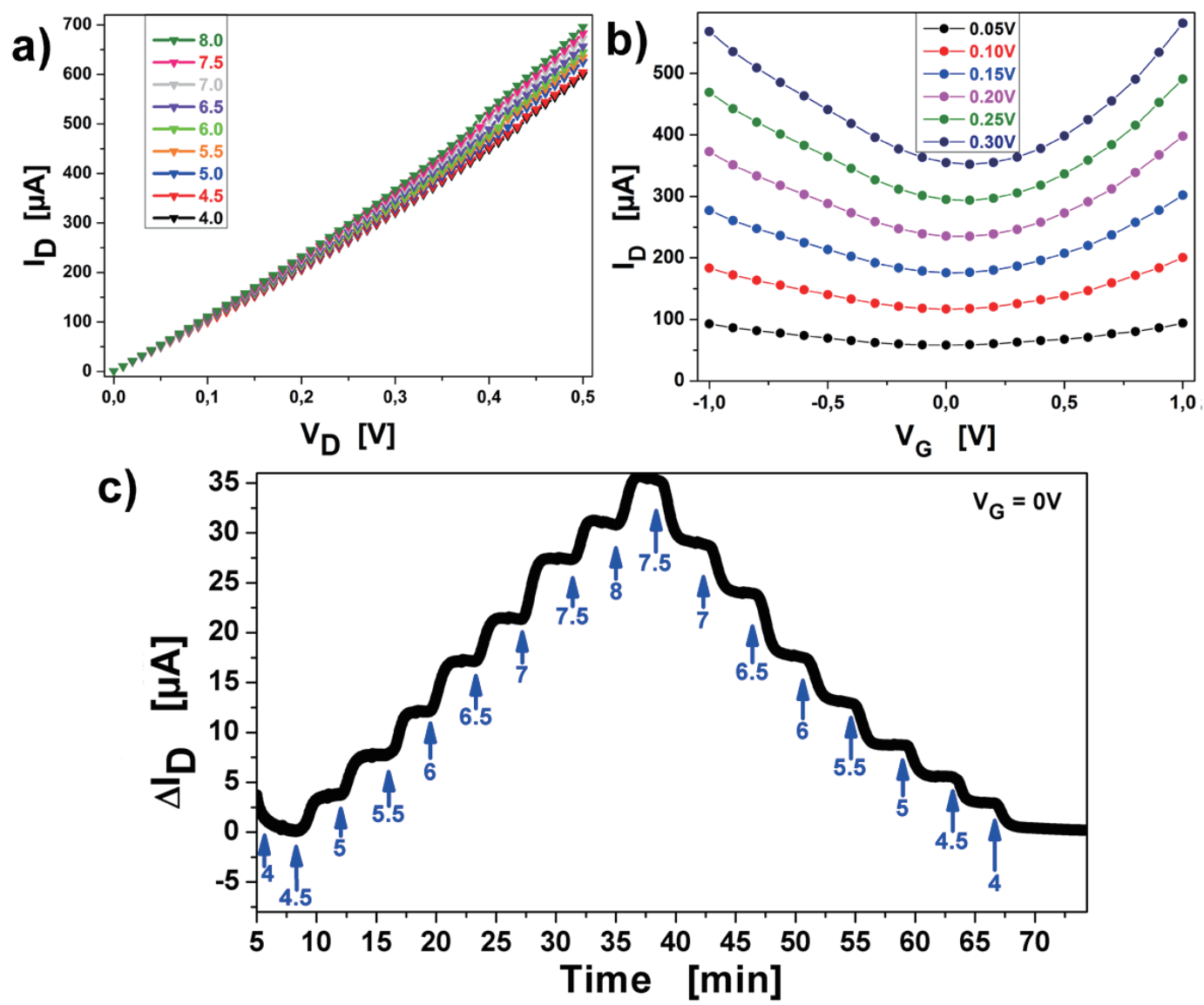

FIG. 2. a) Output characteristics $I_{\mathrm{d}}-V_{\mathrm{d}}$ (drain current-drain voltage) of the transistor device under different applied voltages. b) The $I_{\mathrm{d}}-V_{\mathrm{g}}$ (drain current-gate voltage) transfer curve of bottom-gated grapheme FET under sweeping $V_{\mathrm{g}}$. c) pH titration curve showing titration steps of 0.1 and 0.15 , starting at $\mathrm{pH} 6$ with increasing $\mathrm{pH}$ until $\mathrm{pH} 7$.

\section{E. pH titration curve measurements}

As a brief demonstration of the sensing capabilities of fabricated rGO FET devices, liquid gated measurements were performed using phosphate buffered saline (PBS) as Gate isolating medium. The dependence to $\mathrm{pH}$ value change of the solution was tested and the response signal correlated to the $\mathrm{pH}$ values. Figure 2 (c) shows a titration curve of buffers with $\mathrm{pH}$ varying from 6 to 7 conducted in liquid-gated configuration using constant Gate voltage of $-100 \mathrm{mV}$ and constant Source-Drain voltage of 200 $\mathrm{mV}$. Reversibility of the response signal was confirmed by backwards titration and the response shows linear behaviour in regards to $\mathrm{pH}$ with a slope of $6.1 \mu \mathrm{A} / \mathrm{pH}$. The noise level was found around $100 \mathrm{nA}$, enabling to distinguish between solution's $\mathrm{pH}$ down to differences of $\mathrm{pH}$ 0.02. Charge carrier mobility of the FETs was found from 10000 to $30000 \mathrm{~cm}^{2} / \mathrm{Vs}[20]$.

\section{F. SECM}

The advantage of the constant distance mode is the decoupling of the electrochemical activity information from the topography [30, 31]. Thus, changes in the recorded tip currents correspond only to changes in the electrochemical activity of the investigated surfaces and the current correlates to the tip position on the surface. In order to demonstrate the imaging capability, the topography and the electrochemical activity of a blank $\mathrm{SiO}_{2} / \mathrm{Si}$ substrate, a GO substrate and a rGO substrate were measured. To verify the functionality and stability of the microelectrode, cyclic voltammetry (CV) measurements were done using standard protocols $[30,31]$ before and after each surface investigation (results are presented in appendix A). A linear baseline was created for each surface investigation, starting with the current corresponding to the applied potential from before and ending with the current from after the measurement.

Figure 3 shows the 2D and the 3D images of tip currents of a blank sample (a, b), a GO-sample (c, d) and a rGO-sample (e, f) measured by constant distance mode. The tip current was measured between 120 and $160 \mathrm{pA}$ for the blank sample, indicating very low electrochemical activity. Silicon dioxide is a non-conductive material and is not able to reduce the oxidized form of the mediator (negative feedback). The GO-sample also shows non-conductive behaviour. As shown in Fig. 3 (d), a tip current of approximately $200 \mathrm{pA}$ was recorded at the beginning of the measurement while during the measurement a continuous and nearly linear increase of tip current up to approximately $400 \mathrm{pA}$ was observed. However, no current peaks are found, indicating a localized electrochemical activity of the surface. The continuous increase of the tip current is caused either by the evaporation of the electrolyte due to the measuring time of 17 hours (increasing the redox mediator concentration over time) or 

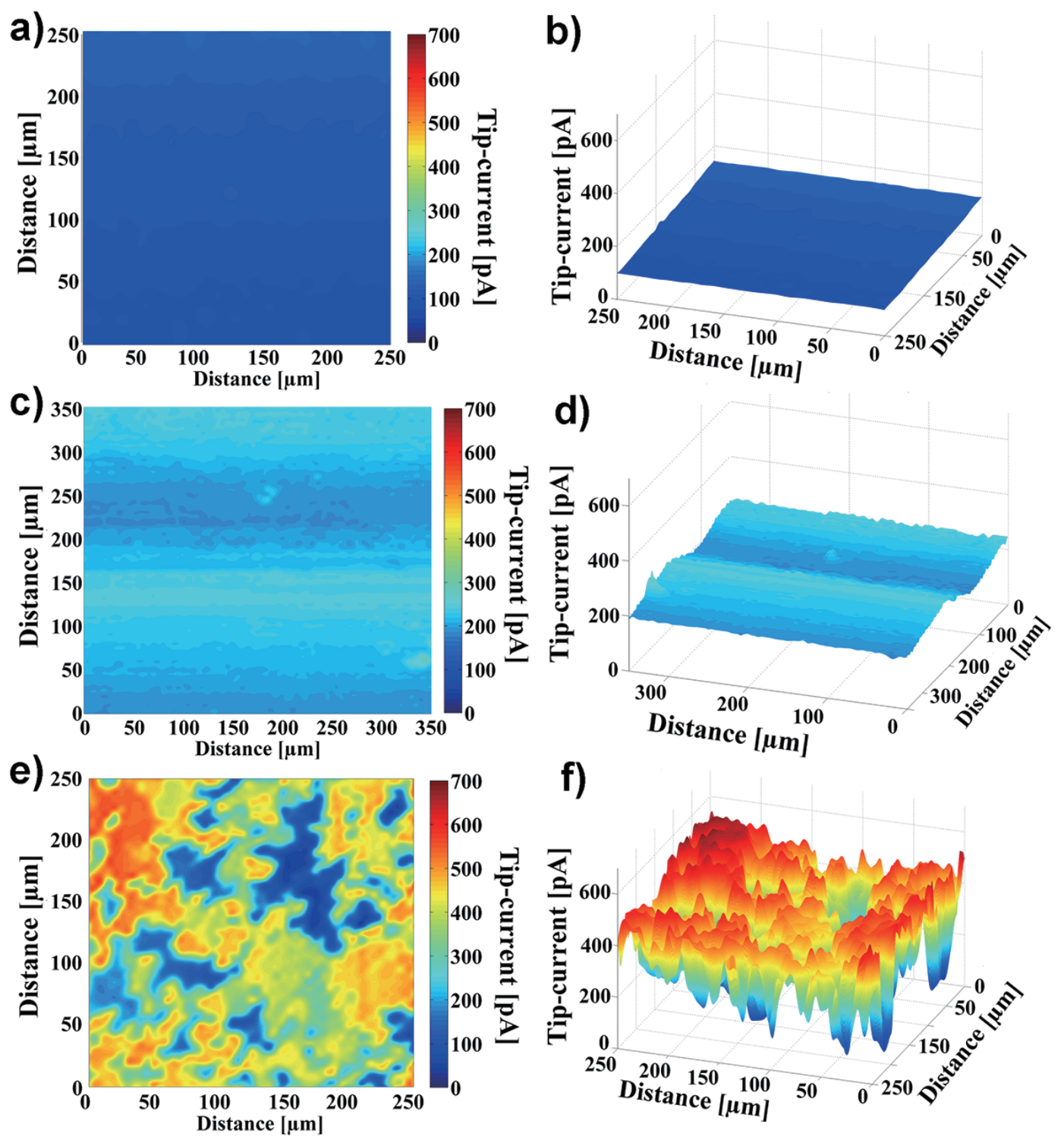

f)

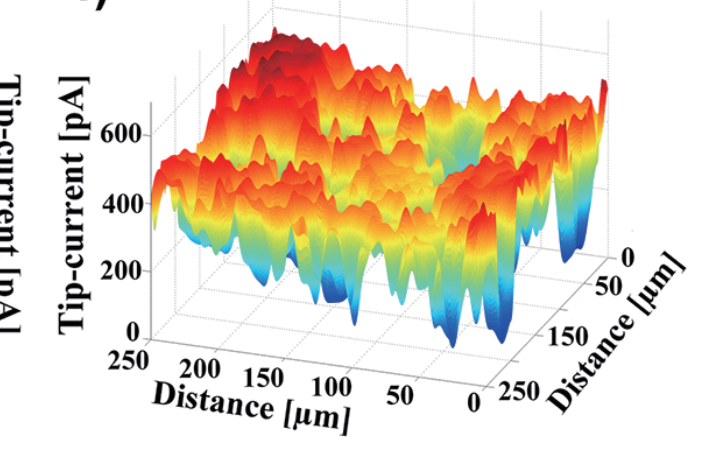

FIG. 3. a) 2D and b) 3D constant distance SECM images of tip-current from blank sample; Tip currents from 120 to 160 pA were measured, thus silicon dioxide is a non-conductive material and is not able to reduce the oxidized form of the mediator (negative feedback). c) 2D and d) 3D constant distance SECM images of tip-current from GO-sample; During the measurement a continuous and nearly linear increase of tip current from 200 pA up to approximately 400 pA could be observed. However, no current peaks were observed, indicating a localized electrochemical activity of the surface. e) $2 \mathrm{D}$ and f) 3D constant distance SECM images of tip-current from rGO-sample; Blue and dark blue areas: electrochemical non-active areas; yellow and red areas: electrochemical active areas (rGO-flakes), since the surface is able to reduce the oxidized form of the redox mediator.

by the shear force unit-change in SF-amplitude signal, resulting in a change of the distance between tip and surface. This effect has to be further investigated, but the increase of the signal was linear over time and corrected by baseline subtraction, yielding maximum tip currents of $300 \mathrm{pA}$. The response after GO deposition did not significantly vary compared to the response of the blank silicon wafer. This result was expected and can be explained due to the insulating properties of GO caused by its primary $\mathrm{sp}^{3}$ hybridization and disrupted $\mathrm{sp}^{2}$ structure [32] The measurements of the tip currents for rGO-samples showed completely different behaviour than GO-samples and blank samples. In electrochemical non-active areas the measured tip-current was found from 150 to $200 \mathrm{pA}$ (dark blue area in 2D and 3D images) - same as the re- sults from the blank sample and the GO-sample. Higher tip-currents about 400-650 pA were measured in large areas on the sample (yellow and red areas in 2D and 3D images), overlapping with the rGO structure observed by standard SEM (Fig. 1 (c)). When the active Pt surface of the tip was positioned completely over a rGO flake, the highest current of $650 \mathrm{pA}$ was measured. If the active $\mathrm{Pt}$ surface covers only a part of the rGO flake, or the flake was smaller than the area of the active Pt surface itself, a lower current was measured accordingly. Depending on the electrochemical properties the current varies from approximately $150 \mathrm{pA}$ over non-electrochemical-active areas to approximately $650 \mathrm{pA}$ over completely electrochemicalactive-areas (rGO-flakes larger than the active $\mathrm{Pt}$ area). It can be concluded that these electrochemical active ar- 


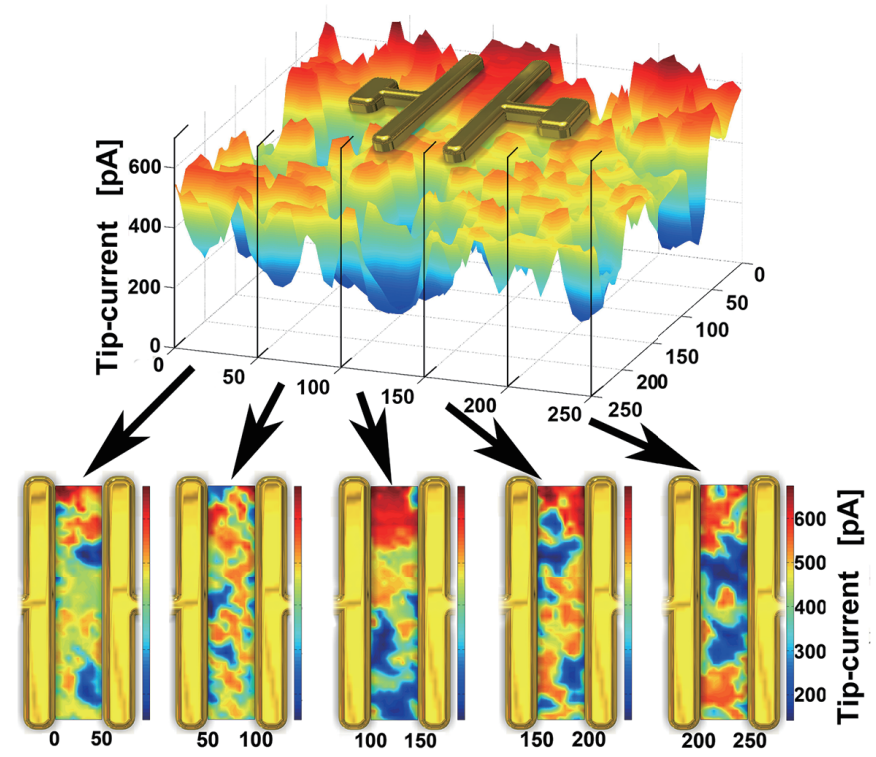

FIG. 4. Five, equal-sized, parallel slices of 3D SECM data of rGO-sample with indicated Au electrodes on the edges of the slices for evaluation of functionality of the rGO-FET; coverage density of slice 1: $79 \%$; slice 2: $74 \%$; slice 3: $64 \%$; slice 4: $67 \%$; slice 5: $57 \%$.

eas are resulting from rGO since the surface is able to reduce the oxidized form of the redox mediator. This result confirms the applicability of SECM for the determination of the rGO surface coverage as well as the rGO reduction success at once for large areas.

\section{G. Evaluation of the functionality of the rGO-FET by means of SECM results}

To evaluate the functionality and reproducibility of the rGO-FETs, which depends on the contact of Source-Drain via $\mathrm{rGO}$, electrodes with a channel width of $50 \mu \mathrm{m}$ were simulated using the SECM data. This approach should reassure the statistical balancing of the rGO distribution in the FET channel using $4 \mathrm{~mm}$ long electrodes [20]. As a visualization of this approach a $3 \mathrm{D}$-presentation of SECM data with graphical illustration of the electrodes and channel contacts is given in the Fig. 4 in the supplemental information. Each slice measures $50 \mu \mathrm{m}$ in width (actual FET channel width). The electrochemically active areas connecting the Source and Drain electrodes provides the electrical contact needed for biosensing applications. In this manner, five channels of the rGO-FET were simulated. The surface areas with a tip current of $\geq 400 \mathrm{pA}$ were defined as electrochemically active, since the measurements of unreduced GO displayed maximum tip currents of $300 \mathrm{pA}$ and these GO areas are found to be non-suitable for rGO-FET biosensors.

The coverage density was calculated via Mathlab (Mathworks, 7.13, R2011b), obtaining following results for the five different slices: $79 \%$ coverage density (slice 1), $74 \%$ (slice 2), $64 \%$ (slice 3 ), $67 \%$ (slice 4 ) and $57 \%$ (slice $5)$. It can be concluded that the rGO coverage density of $68 \pm 7 \%$ provides a very high probability of rGO electrical contact between in the FET channel, rendering the provided approach using long FET channels to balance to statistical variation of GO distribution as a reproducible and applicable method.

\section{CONCLUSION}

GO from wet-chemical synthesis was applied on $\mathrm{SiO}_{2} / \mathrm{Si}$-substrates with the downside of random GO distribution. The samples were then reduced to rGO and coated with electrodes to fabricate FETs. SEM provided the morphology of the samples, while Raman and XPS proved the success of reduction. Comparatively SECM operated in the constant distance mode was used to investigate and evaluate the morphology and the reduction success of the rGO, yielding all needed surface characterization at once. It was found that the calculated degree of rGO coverage from SECM data was $(68 \pm 7) \%$, demonstrating that the electronic conductivity in the FET channel is sufficient and reproducible in spite of the random rGO distribution when using the presented approach. SECM is demonstrated to be a very suitable and simple technique to fully characterize the suitability of rGO surfaces for the application in FETs for biosensing.

\section{ACKNOWLEDGMENTS}

The work at CEST was supported within the COMET program by the Austrian Research Promotion Agency (FFG) and the government of Lower Austria. Special thanks to Heka Elektronik Dr. Schulze GmbH for providing the SECM device, to Christoph Steininger for data analysis and Johannes Srajer for graphical illustration.

\section{APPENDIX}

Description on the setup of scanning electrochemical microscopy and details of characterization of microelectrode are available in Supplementary materials (https://www.jstage.jst.go. jp/article/ejssnt/13/0/13_366/_article/supplement).
[1] M. Pumera, A. Ambrosi, A. Bonanni, E. L. K. Chng, and H. L. Poh, TRAC-Trend. Anal. Chem. 29, 954 (2010).

[2] A. K. Geim and K. S. Novoselov, Nat. Mater. 6, 183 (2007).
[3] K. S. Novoselov, A. K. Geim, S. V. Morozov, D. Jiang, M. I. Katsnelson, I. V. Grigorieva, S. V. Dubonos, and A. A. Firsov, Nature 438, 197 (2005).

[4] Y. Shao, J. Wang, H. Wu, J. Liu, I. A. Aksay, and Y. Lin, Electroanal. 22, 1027 (2010). 
[5] K. V. Sreekanth, S. Zeng, J. Shang, K. T. Yong, and T. Yu, Sci. Rep. 2, 737 (2012).

[6] X. Li, H. Zhu, K. Wang, A. Cao, J. Wei, C. Li, Y. Jia, Z. Li, X. Li, and D. Wu, Adv. Mater. 22, 2743 (2010).

[7] K. Yang, S. Zhang, G. Zhang, X. Sun, S. T. Lee, and Z. Liu, Nano Lett. 10, 3318 (2010).

[8] R. Nandkishore, L. S. Levitov, and A. V. Chubukov, Nat. Phys. 8, 158 (2012).

[9] H. C. A. Becerril, J. Mao, Z. Liu, R. M. Stoltenberg, Z. Bao, and Y. Chen, ACS Nano 2, 463 (2008).

[10] D. Wei, Y. Liu, Y. Wang, H. Zhang, L. Huang, and G. Yu, Nano Lett. 9, 1752 (2009).

[11] J. Molina, J. Fernández, J. C. Inés, A. I. del Río, J. Bonastre, and F. Cases, Electrochim. Acta 93, 44 (2013).

[12] J. Molina, J. Fernández, J. C. Inés, A. I. del Río, J. Bonastre, F. Cases, Appl. Surf. Sci. 279, 46 (2013).

[13] R. M. Souto, J. J. Santana, L. Fernández-Mérida, S. González, Electrochim. Acta 56, 9596 (2011).

[14] Y. Yin, L. Niu, M. Lu, W. Guo, and S. Chen, Appl. Surf. Sci. 255, 9193 (2009).

[15] P. Sun, F. O. Laforge, and M. V. Mirkin, Phys. Chem. Chem. Phys. 9, 802 (2007).

[16] S. E. Pust, W. Maier, and G. Wittstock, Z. Phys. Chem. 10, 1463 (2008).

[17] C. Kranz, G. Wittstock, H. Wohlschläger, and W. Schuhmann, Electrochim. Acta 42, 3105 (1997).

[18] M. V. Mirkin, and B. R. Horrocks, Anal. Chim. Acta 406, 119 (2000).

[19] B. Ballesteros Katemann, A. Schulte, and W. Schuhmann, Chem.-Eur. J. 9, 2025 (2003).

[20] C. Reiner-Rozman, M. Larisika, C. Nowak, and W. Knoll, Biosens. Bioelectron. 70, 21 (2015).
[21] A. J. Bard, and M. V. Mirkin, Scanning Electrochemical Microscopy (CRC, Boca Raton, 2012).

[22] M. Larisika, J. Huang, A. Tok, W. Knoll, and C. Nowak, Mater. Chem. Phys. 136, 304 (2012).

[23] M. Etienne, A. Schulte, S. Mann, G. Jordan, I. D. Dietzel, and W. Schuhmann, Anal. Chem. 76, 3682 (2004).

[24] B. Ballesteros Katemann, A. Schulte, and W. Schuhmann, Electroanal. 16, 60-65 (2004).

[25] A. Hengstenberg, C. Kranz, and W. Schuhmann, Chem.Eur. J. 6, 1547 (2000).

[26] A. C. Ferrari, J. C. Meyer, V. Scardaci, C. Casiraghi, M. Lazzeri, F. Mauri, S. Piscanec, D. Jiang, K. S. Novoselov, S. Roth, and A. K. Geim, Phys. Rev. Lett. 97, 187401 (2006).

[27] J. Huang, M. Larisika, W. H. D. Fam, Q. He, M. A. Nimmo, C. Nowak, and I. Y. A. Tok, Nanoscale 5, 2945 (2013).

[28] S. Stankovich, R. D. Piner, X. Chen, N. Wu, S. T. Nguyen, and R. S. Ruoff, J. Mater. Chem. 16, 155 (2006).

[29] S. Gilje, S. Han, M. Wang, K. L. Wang, and R. B. Kaner, Nano Lett. 7, 3394 (2007).

[30] M. A. Mezour, M. Morin, and J. Mauzeroll, Anal. Chem. 83, 2378 (2011).

[31] B. Ballesteros Katemann, and W. Schuhmann, Electroanal. 14, 22 (2002).

[32] D. R. Dreyer, S. Park, C. W. Bielawski, and R. S. Ruoff, Chem. Soc. Rev. 39, 228 (2010).

[33] C. Cougnon, K. Bauer-Espindola, D. S. Fabre, and J. Mauzeroll, Anal. Chem. 81, 3654 (2009).

[34] C. Y. Su, Y. Xu, W. Zhang, J. Zhao, X. Tang, C. H. Tsai, and L. J. Li, Chem. Mater. 21, 5674 (2009).

[35] Y. Xu, H. Bai, G. Lu, C. Li, and G. Shi, J. Am. Chem. Soc. 130, 5856 (2008).

[36] G. Eda, G. Fanchini, M. Chhowalla, Nat. Nanotechnol. 3, 270 (2008). 